\title{
Morfologia e morfometria dos tipos de fibras do músculo reto abdominal de cadelas (Canis familiaris) durante a gestação
}

\author{
Maila Karina Mattos de BRITO'1 \\ Colombo Guerra CARVALHO \\ JUNIOR ${ }^{2}$ \\ Alexandre DAL $\mathrm{PAI}^{3}$ \\ Vitalino DAL PAI ${ }^{2}$ \\ José Alberto GIACOMETTI ${ }^{1}$
}

Correspondência para:

R: Zico Leite, 98 - Chavantes - SP, CEP: 18.970-000.

e-mail: mailakarina@hotmail.com

Recebido para publicação: 31/08/2005 Aprovado para publicação: 27/03/2007

\author{
1 - Faculdade de Ciências e Tecnologia, Universidade Estadual Paulista, \\ Presidente Prudente - SP \\ 2 - Universidade do Oeste Paulista, Presidente Prudente - SP \\ 3 - Faculdade de Tecnologia, Botucatu - SP
}

\section{Resumo}

Neste trabalho foram avaliadas as alterações morfológicas e o grau de hipertrofia das fibras de contração lentas (tipo I) e rápidas (tipo II) do músculo reto abdominal de cadelas adultas nas fases: prégestacional, 30 e 60 dias de gestação e 30 dias pós-parto (pp). Em todas essas fases, sob anestesia, foram removidas biópsias musculares, as quais foram congeladas. Cortes histológicos foram obtidos em um micrótomo e corados com hematoxilina e eosina (HE) para a avaliação da morfologia. Outros cortes foram submetidos à ATPase miofibrilar (m-ATPase), após pré-incubação em meio alcalino $(\mathrm{pH}=10,4)$ e em meio ácido $(\mathrm{pH}=4,4)$, visando à identificação das fibras tipo I e II. Nas cadelas virgens, o tecido muscular revelou-se constituído por fibras com diferentes diâmetros, com contornos poligonais, tendo um ou mais núcleos periféricos. Estas características foram similares nos animais com 30 dias de gestação. Aos 60 dias, ao lado de fibras com aparência normal, observaram-se fibras com contornos mais polimórficos e outras fibras de diâmetros muito pequenos. Aos 30 dias pp, as características do tecido foram similares às dos animais em fase pré-gestacional. Em todas as fases estudadas, os diâmetros fibras tipo II foram maiores do que as do tipo I. Aos 30 dias observou-se a redução significativa dos diâmetros de ambos os tipos de fibras e aumento significativo aos 60 dias de gestação. Durante a gestação, o estímulo crônico, representado pela expansão da parede abdominal, promoveu alterações da morfologia e morfometria das fibras.
Palavras-chave: Músculo reto abdominal. Morfometria. Fibras musculares tipo I e II. Gestação. Cadelas.

\section{Introdução}

Conforme revelado pelos métodos de histoquímica enzimática, o tecido muscular estriado esquelético dos mamíferos está constituído por dois tipos básicos de fibras com diferentes características morfológicas, metabólicas e contráteis ${ }^{1,2}$. Com base na reação m-ATPase as fibras são classificadas em tipo I, de contração lenta e metabolismo oxidativo, e tipo II, de contração rápida. Estas, por sua vez, são divididas em tipo IIA, com metabolismo oxidativo-glicolítico e IIB, com metabolismo glicolítico ${ }^{3,4,5,6}$. As fibras tipo II são geralmente maiores do que as do tipo I, tendo maior capacidade para gerar tensão máxima ${ }^{7}$. A freqüência e a distribuição dos tipos de fibras nos diferentes músculos é variável entre as espécies, raças, indivíduos, idade, sexo, grupo muscular, a atividade física, o desuso, a desnutrição ${ }^{8}$, a desnervação ${ }^{9}$ e o estresse fisiológico crônico ${ }^{10}$. Neste campo da investigação a utilização de métodos morfológicos e histoquímicos tem-se revelado importante na avaliação da fisiologia muscular, desde $1970^{3}$.

Nos mamíferos, o aumento do conteúdo abdominal durante a gestação 
representa um estímulo fisiológico crônico imposto à musculatura da parede. A intensidade e a duração deste estímulo promove alterações nas características morfofuncionais das fibras. Uma vez que o estímulo é variável em função da espécie animal, do período gestacional, do número e tamanho dos fetos, bem como das estruturas associadas ${ }^{11,12,13,14,15,16,17}$, os efeitos são também variáveis e objeto de numerosas investigações. Essas investigações são realizadas através de biópsias musculares, as quais são utilizadas também para diagnósticos de diferentes patologias ${ }^{18,19,20}$.

Uma vez que as informações da literatura sobre a morfologia, morfometria e a histoquímica dos tipos de fibras em músculos abdominais de cães ao longo da gestação são ainda escassas, a presente pesquisa tem por finalidade avaliar no músculo reto abdominal, as características morfológicas e o grau de hipertrofia das fibras dos tipos I e II em diferentes estágios de gestação.

\section{Material e Método}

Foram utilizadas cinco cadelas (Canis familiaris), sem raça definida, virgens, com idade e peso corporal que variaram entre 1 e 1,5 anos e de 10,9 a 12,5 kg, respectivamente. Os animais foram mantidos em gaiolas de 1,5 × 2,0 × 1,5 m no canil da Universidade do Oeste Paulista (UNOESTE), Presidente Prudente, SP, com ração peletizada e água ad libitum.

Decorridos 20 dias de adaptação, os animais foram anestesiados mediante injeções de acepromazina $(0,10 \mathrm{mg} / \mathrm{kg})$ e após 15 minutos quetamina $(15 \mathrm{mg} / \mathrm{kg})$, ambas por via intramuscular. A seguir, da região pré-umbilical do músculo reto do abdome foram removidas amostras de tecido, as quais foram imersas em n-Hexana a $-70{ }^{\circ} \mathrm{C}$, durante dois minutos.

A primeira biópsia colhida em fase pré-gestacional representou o grupo controle. A seguir, os animais foram dispostos em dois grupos experimentais, oriundos de aleatorização, que foram mantidos juntos com um macho, em gaiolas apropriadas. Após 20 dias, as cadelas tiveram o teste de prenhez positivo. No $30^{\circ}$ dia após o diagnóstico, sob anestesia, foi efetuada a $2^{a}$ coleta de fragmentos da região préumbilical do músculo em estudo. Mantido o método de coleta, foi realizada a $3^{\text {a }}$ coleta no $60^{\circ}$ dia de gestação e a $4^{a}$ coleta, no $30^{\circ}$ dia pós-parto (pp). Usando um micrótomo criostato $\left(-20{ }^{\circ} \mathrm{C}\right)$ foram obtidos cortes histológicos ( $8 \mu \mathrm{m}$ de espessura), transversais ao maior eixo das fibras. Para a avaliação da morfologia, os cortes foram corados pela hematoxilina e eosina (HE). Visando à identificação das fibras de contração lenta (tipo I) e as de contração rápida (tipo II = IIA + IIB), cortes histológicos foram submetidos á reação ATPase miofibrilar (mATPase), após pré-incubação em pH 4,4 e em pH 10,421.

Para a avaliação do grau de hipertrofia, as lâminas foram inicialmente visualizadas e digitalizadas, utilizando-se uma câmara digital, acoplada a um microscópio Leica. A seguir, sob ampliação de $50 \mathrm{X}$, em vários campos microscópicos, foram amostradas, para cada animal, 100 fibras de cada tipo, seguindo-se a análise morfométrica. A hipertrofia das fibras foi avaliada pelo diâmetro médio $(\mathrm{mm})$ da secção transversal dessas fibras, utilizando o software Image-ProPlus, versão 4.5 , que possibilitou a segmentação manual das imagens das fibras, e permitiu o calculo do perímetro através da medida do comprimento da curva que contorna cada fibra. O diâmetro das fibras foi obtido pela posição do centróide da seção transversal da fibra.

As comparações dos diâmetros médios das fibras I e II foram feitas por ANOVA, com significância de 5\% dentro de cada período gestacional e entre os períodos (Tabela 1).

\section{Resultados e Discussão}

O tecido muscular das cadelas virgens revelou-se constituído por fibras de diferentes diâmetros, tendo contornos poligonais e com um ou mais núcleos 
periféricos. Estas características mostraramse similares nos animais com 30 dias de gestação (Figura 1A), tendo as fibras, porém, menor diâmetro. Aos 60 dias, ao lado de fibras com aparência normal, observaramse algumas fibras com contornos polimórficos, além de aumento do número de fibras com diâmetros muito pequenos (Figura 1C).

$\mathrm{Na}$ reatividade da m-ATPase miofibrilar, após pré-incubação em meio ácido $\mathrm{pH}$ 4,4 o músculo reto do abdome da cadela revelou-se composto por três tipos de fibras: tipo I, contendo miosina de contração lenta, tipo IIA e IIB, com miosina de contração rápida. A freqüência de fibras IIB revelou-se muito baixa (Figuras 1B e 1D). Após pré-incubação em pH 10,4, os tipos de fibras revelaram reversão da atividade (Figura 1C). Além disso, as fibras revelaram um padrão de distribuição em "mosaico"
(Figura 1D).

O diâmetro das fibras dos tipos I e II, nos períodos 0, 30, 60 dias de gestação e 30 dias (pp), apresentaram variações significativas. Na presente pesquisa, as fibras tipo II, devido ao seu teor de enzimas da via metabólica oxidativa e metabolismo glicolítico inferior à do tipo I, mostraram maior aumento no diâmetro, especialmente aos 60 dias de gestação (Tabela 1). Observase nas figuras 2 e 3 a distribuição da freqüência das fibras tipo I e II respectivamente, para todos os períodos estudados.

Durante a gestação a musculatura abdominal se adapta ao estresse provocado pelo aumento do peso do conteúdo intrauterino. Segundo Martin ${ }^{11}$, um dos efeitos produzidos pela sobrecarga imposta à musculatura abdominal consiste no estiramento das fibras da musculatura abdominal.

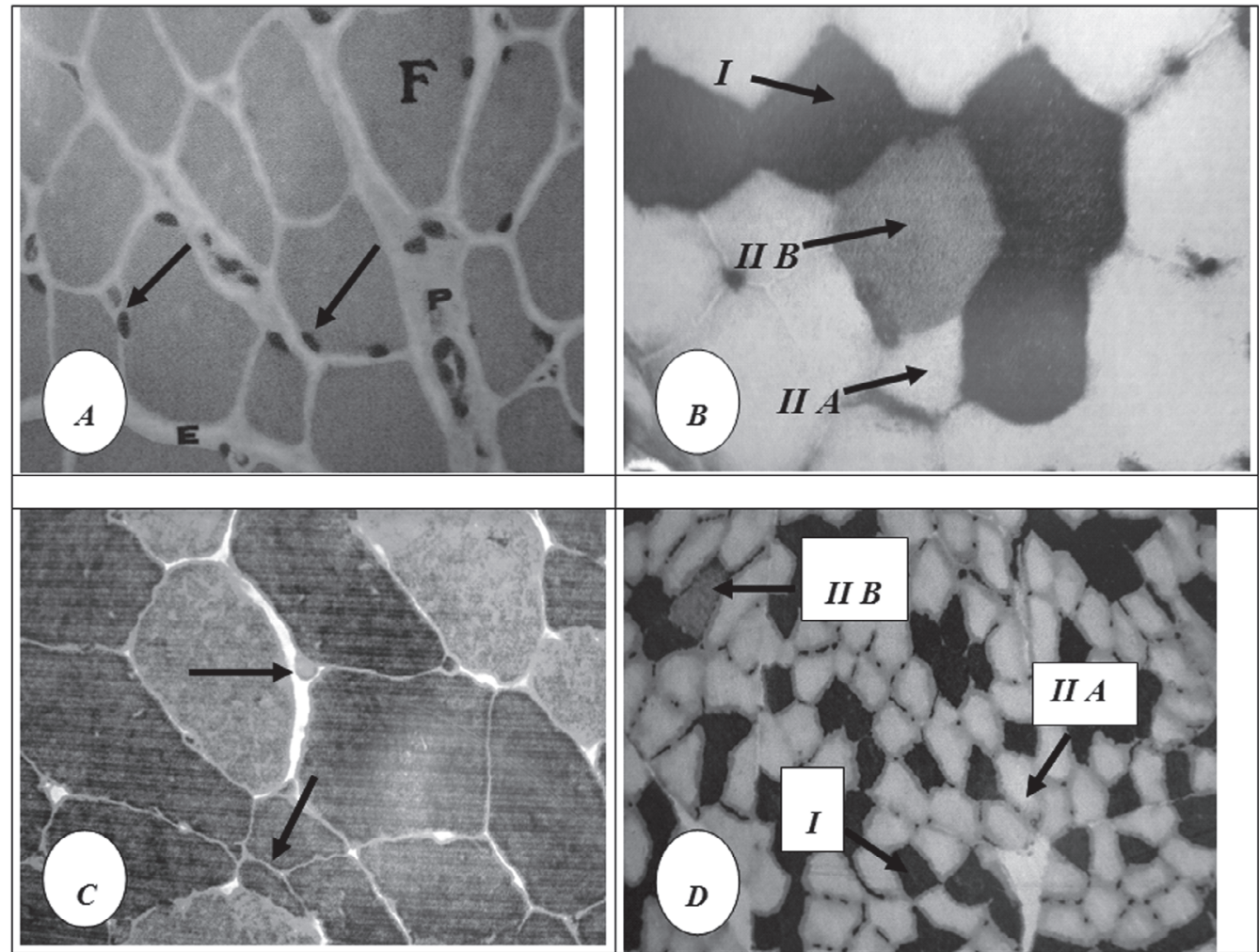

Figura 1 - Cortes transversais do músculo reto do abdome de cadela (A-D). A) Fibras com diferentes contornos e diâmetros (F); Perimísio(P); Endomísio (e); núcleos periféricos (setas). HE. 50X. B) Fibras de contração lenta (I) e de contração rápida (IIA e IIB), m-ATPase, (pH 4,4). 50X. C) Fibras mais claras (I); fibras escuras (IIA e IIB); fibras com diâmetro pequeno (setas), m-ATPase, (pH 10,4). 50X. D) Fibras I, IIA e IIB com distribuição "em mosaico", m-ATPase, (pH 4,4), $20 \mathrm{X}$ 
Tabela 1 - Comparação entre as médias dos diâmetros mínimos $(\mathrm{mm})$ e desvio padrão das fibras tipo I e II do músculo reto do abdome dos animais, apresentando a significância estatística para os períodos de gestação. [Número de fibras amostradas (500), $\alpha=0,05$.

\begin{tabular}{ccc}
\hline FASES DA & FIBRAS TIPO I & FIBRAS TIPO II \\
GESTAÇÃO & (FOG) & (FOG + FG) \\
\hline $\mathbf{0}$ & $35,0 \pm 8,7^{\mathrm{a}}$ & $41,0 \pm 9,7^{\mathrm{a}}$ \\
$\mathbf{3 0}$ & $33,0^{\mathrm{a}} \pm 7,1^{\mathrm{a}}$ & $36,0 \pm 6,8^{\mathrm{a}}$ \\
$\mathbf{6 0}$ & $39,0^{\mathrm{a}} \pm 8,2^{\mathrm{a}}$ & $42,5 \pm 8,3^{\mathrm{a}}$ \\
$\mathbf{3 0}$ ap & $39,4 \pm 5,7^{\mathrm{b}}$ & $42,4 \pm 7,3^{\mathrm{b}}$ \\
\end{tabular}

a) médias significativamente diferentes; b) médias NÃO significativamente diferentes.

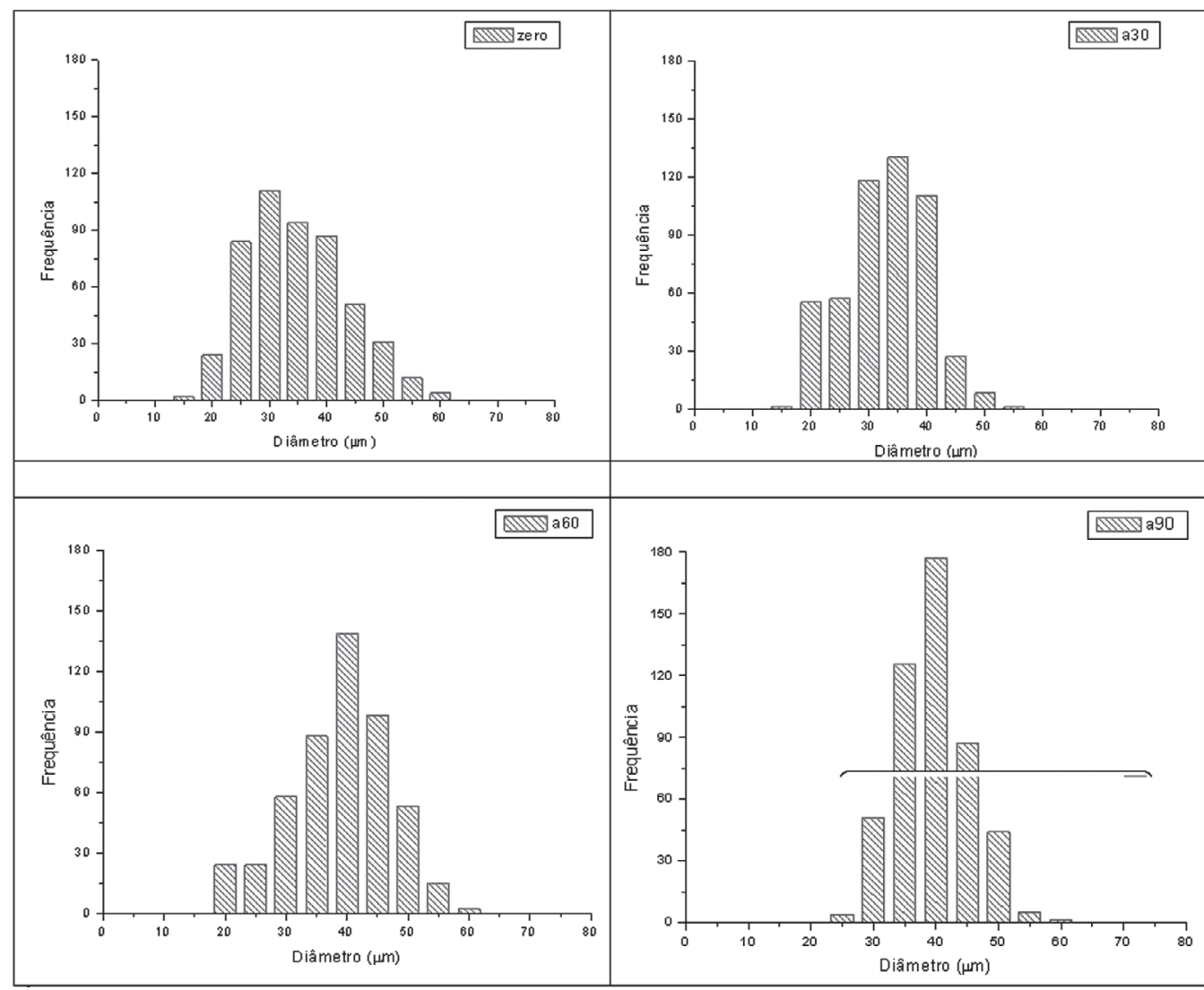

Figura 2 - Freqüência das fibras tipo I

O significativo aumento do diâmetro das fibras observado no período de 30 a 60 dias de gestação, por outro lado, parece estar intimamente associado ao aumento do peso do conteúdo intra-uterino. Assim, os resultados observados revelam que a sobrecarga exercida sobre a parede abdominal, além de promover aumento no diâmetro das fibras, propiciam o aparecimento de alterações morfológicas, 


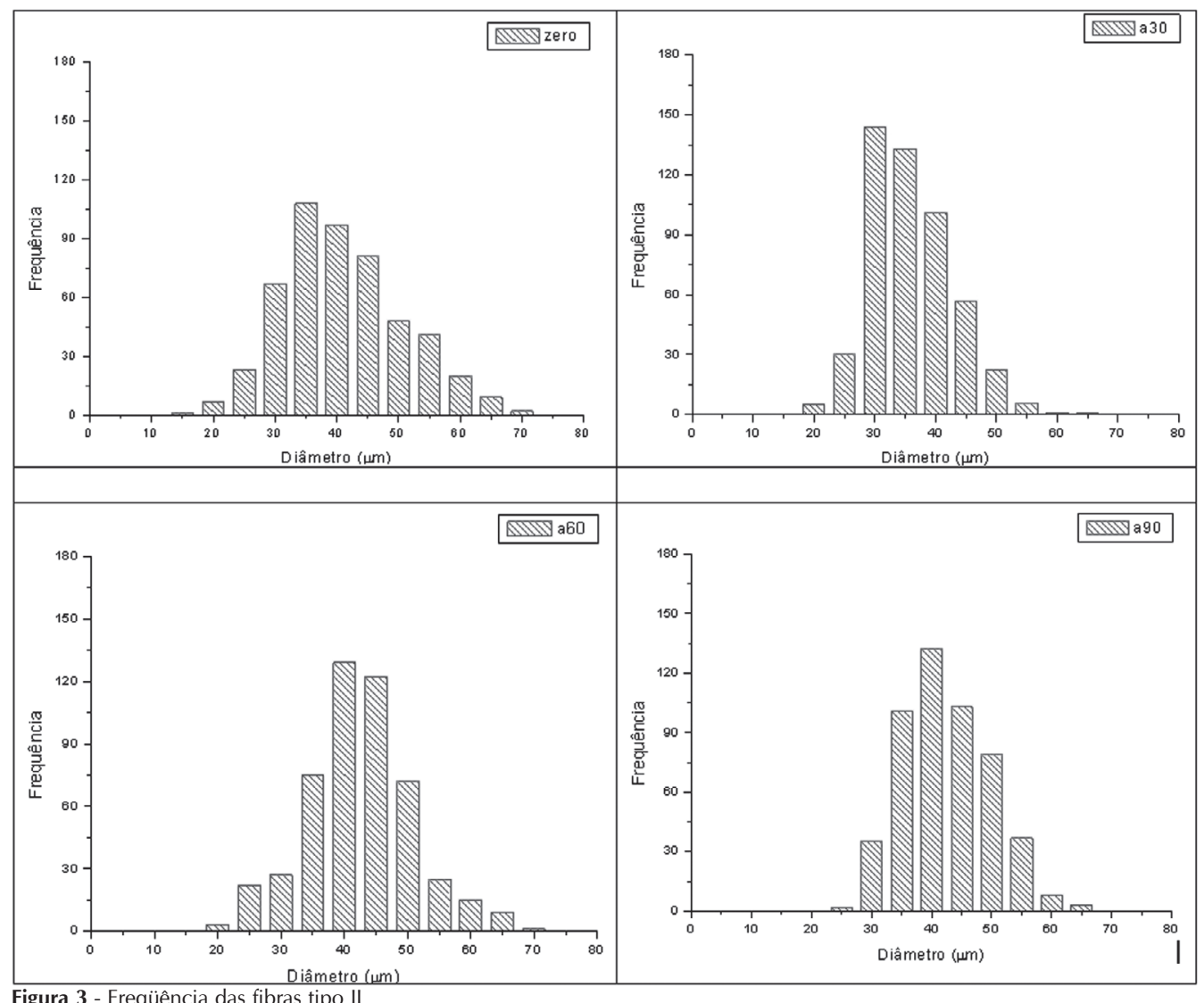

Figura 3 - Freqüência das fibras tipo II

representadas pelo maior número de fibras com diâmetro pequeno.

O músculo reto do abdome do cão possui como característica marcante, coloração vermelha intensa, sendo isto conseqüência de sua elevada concentração de fibras I e IIA ${ }^{22}$. Estas fibras são também referidas como vermelhas, com metabolismo oxidativo. No entanto, as fibras IIA, de contração rápida, possuem atividade metabólica oxidativa um pouco menos intensa, podendo, assim, assumir diâmetro maior.

Além disso, os resultados revelaram que, tanto nos diferentes períodos da prégestação, quanto aos 60 dias houve aumento significativo do diâmetro médio das fibras I quando comparadas com as fibras tipo II, porém no período de 60 dias de gestação e 30 dias (pp), não houve alteração significativa do diâmetro das fibras, uma vez que já havia cessado o estímulo gestacional sobre o músculo reto abdominal.
De acordo com a literatura, devido à elevada atividade metabólica oxidativa, as fibras vermelhas do tipo I são adaptadas a pequeno diâmetro, tendo assim, facilitadas as trocas metabólicas, a oxigenação e a remoção de resíduos metabólicos. Já, as fibras com metabolismo glicolítico (tipo IIB), que são adaptadas às baixas tensões de oxigênio e de trocas metabólicas, possuem maior habilidade de hipertrofia ${ }^{21,23,24}$.

$\mathrm{Na}$ presente pesquisa, o diâmetro dos tipos de fibras I e II foi avaliado a partir das preparações coradas pela técnica m-ATPase, precedida de incubação em $\mathrm{pH}=4,4$ sendo evidenciados três tipos de fibras: I e IIA e IIB. No entanto, uma vez que, conforme estudo recente, a freqüência de fibras IIB é de $1,8 \%{ }^{22}$, para efeito de quantificação das fibras de contração rápida, considerou-se o conjunto das fibras IIA e IIB. Segundo Kurlberg $^{25}$, nos mamíferos, o aumento do diâmetro das fibras da musculatura 
abdominal observado durante o período gestacional é atribuído à combinação dos fatores estiramento e sobrecarga, contribuindo, assim, para a manutenção do tônus muscular e resistência à fadiga. Ao que parece, a duração do estímulo é fundamental. Em ratas, 21 dias de gestação não provocaram alterações nas freqüências dos tipos de fibras do músculo reto do abdome, porém a hipertrofia das fibras do tipo I, foi maior na segunda metade do período de gestação $^{11}$.

\section{Conclusão}

Durante a gestação, as fibras I e IIA do músculo reto abdominal tiveram seu diâmetro significativamente aumentado, tendo sido maior no período do parto. Nesta fase, o tecido revelou maior número de fibras pequenas, sendo isto sugestivo de processo miogênico. Além do estiramento das fibras, a hipertrofia é resultante da sobrecarga imposta ao músculo pelo peso dos fetos e das estruturas associadas. A redução no diâmetro das fibras tipos I e II observada no período de 0 a 30 dias de gestação parece estar associada a adaptação das fibras à elevada atividade metabólica oxidativa. A morfometria representa um método seguro na avaliação do grau de acometimento seletivo sobre os tipos de fibras, em especial nos músculos envolvidos com a fisiologia da gestação.

\section{Morphologic and morphometry of rectus abdominis muscle fiber types of the during the gestation dog (Canis familiaris)}

\begin{abstract}
The present paper deals with the evaluation of morphological and morphometric alterations of slow-twist (I) and fast-twitch (II) fibers of rectus abdominis muscle of adult female dog during pregestational phase, at 30 and 60 days of pregnancy and at 30 days after the parturition. At the every phases, using the open biopsy technique, muscle samples were collected. The samples were plunged. Histological sections were cut in a microtome. For general morphology, some sections were stained with HE. Subsequent sections were reacted for myofibrillar ATPase (m-ATPase), after alkaline $(\mathrm{pH}$ 10.4) and acid $(\mathrm{pH} 4.4)$ pre-incubations, in order to identificate type I and II fibers. In the pre-gestational phase, muscle tissue revealed to be composed by fibers with different diameters, presenting polygonal outlines and one or more periphery nuclei. At 30 days of pregnancy, muscle fiber characteristics were similar. At 60 days, in addition to the existence of normal fibers, polymorphic and small diameter fibers were frequent. At 30 days after the parturation, the morphology of muscle fiber were similar to that observed in the pre-gestational phase. In the four phases, type II fiber diameters were lager than type I. The diameters of both fiber types showed a significant reduction in the 30 days phase and a significative increasing at 60 days. The expansion of the abdominal wall during the pregnancy represents a chronic stimulus, induced changes in the morphology and in the fiber type diameters.
\end{abstract}

\section{Key words:}

Retus abdominis muscle. Morphometry.

Fiber type I and II.

Gestation.

Female dog.

Agradecimentos à FAPESP e a Dra. Ana Maria Osório por ter cedido o microscópio. Bolsa de Iniciação Científica, FAPESP, Proc. No. 02/10320-8 


\section{Referências}

1 YELLIN, H.; GUTH, L. The histochemical classification of muscle fibers. Exp. Neurol., v. 26, p. 424-432, 1970.

2 PETTER, J. B.; BARNARD, R. J.; EDGERTON, V. R.; GILLEPSIE, C. A.; STEMPEL, K. E. Metabolic profiles of the three fiber types of skeletal muscle in guinea pig and rabbit. Biochemistry, v. 11, p. 2627-2637, 1972.

3 BROOKE, M. H.; KAISER, K. K. Muscle fiber types: How many and what kind? Arch. Neurol., v. 23, p. 369379,1970

4 LOPEZ-RIVERO, J. L. Características histoquímicas, bioquímicas e morfológicas del musculo esqueletico del equino. Agro Ciencias., v. 9, p. 113-131, 1993b.

5 LOPES-RIVERO, J. L.; DIZ, A. M.; MONTEVERDE, J. G.; AGUERRA E. Intramuscular distribuition of fibre types in the gluteus medius of the horse: A histochemical analysis. Anat. Histol. Embryol., v. 22, p. 233-240, 1993b.

6 LOPES-RIVERO, J. L.; AGUERRA, E.; VIVO, J.; DIZ, A. M.; MIRO F. Histochemical and morphometrical study of the gluteal muscle in Arabian horses. Eq. Vet Sci., v. 10, n.2, p. 144-148, 1990.

7 GARNETT, R. A. F.; O'DONOVAN, M. J.; STHEPHENS, J. A.; TAYLOR, A. Motor unit organization of human medial gastrocnemius. J. Phisiol., v. 287, p. 33-43, 1979.

8 TOMANEK, R. J.; COOPER, R. R. Ultrastructural changes in tenotomized fast and slow twitch muscle fibers. J. Anat., v. 113, p. 409-424, 1972.

9 ENGEL, W.; KING, M.; BROOKE, H.; NELSON, P. G. Histochemical studies of denervated and tenotomized cat muscle: Illustrating the difficulties in relating experimental animal conditions to human neuromuscular diseases. Ann. N.Y.Sci., v. 138, p. 160185, 1966.

10 BROWN, D. Experimental studies pertaining to hypertrophy, regeneration and degeneration. Res. Pub. Assoc. Res. Nerv Mental Dis., v. 38, p. 147-196,1960.

11 MARTIN, W. D. A study of the effect of pregnancy on the muscle fibers of the rectus abdominis muscle of the rat. Anat. Rec., v. 195, p. 455-462, 1979.

12 LALATTA, C. G.; BARAZZONI, A. M.; LUCCHI, M. L.; BORTOLAMI, R. Histochemical Types and Sizes of Fibers in the Rectus Abdominis Muscle of Guinea Pig: Adaptive Response to Pregnancy. Anat. Rec., v. 217, p. 23-29, 1987a
13 LALATTA, C. G.; BARAZZONI, A. M.; LUCCHI, M. L.; BORTOLAMI, R. Effeti della gravidenza normale e prolungata artificialmente sul muscolo reto dell'abdome nella coniglia. In: CONGRESSO DELLA SOCIETÀ ITALIANA DI ANATOMIA, 42., 1987. Siena. Proceedings... Siena: [s.n.], 1987b. p. 181.

14 GURTLER, H.; KETZ, H. A.; KOLB, E.; SCHROEDER, L.; SEIDER, H. 4.ed. Fisiologia veterinária. Rio de Janeiro: Guanabara Koogan. 1987. 611 p.

15 LALATTA, C. G.; BARAZZONI, A. M.; LUCCHI, M. L.; BORTOLAMI, R. Fiber sizes and histochemical characteristics of the rectus abdominis muscle of the rabbit under conditions of pregnancy and mechanically induced stress. Anat. Rec., v. 222, p. 136-144, 1988.

16 SWENSON, M. J.; REECE, W. O.; DUKES, H. H. Dukes fisiologia dos animais domésticos. 11.ed. Rio de Janeiro: Guanabara Koogan, 1996. 856 p.

17 CUNNINGHAM, J. G. Tratado de fisiologia veterinária. 2. ed. Rio de Janeiro: Guanabara Koogan, 1999. $527 \mathrm{p}$.

18 WETTERMAN, C. A.; HARKIN, K. R.; CASH, W. C. NIETFIELD, J. C.; SHELTON, G. D. Hypertrophic muscular dystrophy in a young dog. J Am Vet Medical Association, v. 216, n. 6, p. 878-881, 2000

19 BILZER T. Diagnostic biopsy of muscular diseases in dogs. Praktische Tierarzt., v. 82, n. 6, p. 424-430, 2001.

20 RAWLINGS, C. A.; DIAMOND, H.; HOWERTH, E. W.; NEUWIRTH, L.; CANALIS, C. Diagnostic quality of percutaneous kidney biopsy specimens obtained with laparoscopy versus ultrasound guidance in dogs. J Am Vet Medical Association, v. 223, n. 3, p. 317-321, 2003.

21 DUBOWITZ, V. Muscle biopsy: a pratical approach. 2nd ed. London: Bailliere Tindall, 1985. 475 p.

22 CARVALHO JUNIOR, C. G. Morfologia, metabolismo e características contrateis dos tipos de fibras do músculo reto do abdome do cão (Canis familiares) durante o ciclo reprodutivo. 2003. $91 \mathrm{f}$. Dissertação (Mestrado em Ciências Fisiológicas) Universidade do Oeste Paulista, Presidente Prudente, 2003.

23 BENDALL, J. R.; VOYOLE, A. A study of the histological changes in the growing muscles of beef animals. J. Food Techn, v. 2, p. 259-283, 1967.

24 DALL PAI, V.; THOMAZ, E.; CURI, P. R. Postnatal growth of skeletal muscle fibers of the rat. Gegenbaurs morphol.Jahrb. , v. 6, p. 827-834, 1984.

25 KURLBERG, E. Histochemical composition, contraction speed and fatigability of rat soleous motors units. J. Neurol.Sci., v. 20, p. 177-198, 1973. 